\title{
Quintessential Inflation
}

\author{
P. J. E. Peebles \\ Joseph Henry Laboratories, Princeton University, and \\ Institute for Advanced Study, Princeton NJ 08544 \\ and \\ A. Vilenkin \\ Department of Physics \\ Tufts University, Medford MA 02155
}

(February 1, 2008)

We present an explicit observationally acceptable model for evolution from inflation to the present epoch under the assumption that the entropy and matter of the familiar universe are from gravitational particle production at the end of inflation. This eliminates the problem of finding a satisfactory coupling of the inflaton and matter fields. Since the inflaton potential $V(\phi)$ may be a monotonic function of the inflaton $\phi$, the inflaton energy could produce an observationally significant effective cosmological constant, as in quintessence.

\section{INTRODUCTION}

A satisfactory inflation model for the very early universe has to account for the entropy in matter - the fields of present-day physics or their predecessors. The usual assumption is that the entropy comes from the decay of the inflaton field or fields $\phi$ whose stress-energy tensor drives inflation. In this scenario the inflaton rolls toward a minimum of its potential where it oscillates, and the oscillations are damped by the production of quanta of fields coupled to $\phi$. The couplings have to be extremely weak, to protect the flat inflaton potential which is necessary for a successful inflation picture, but at the same time strong enough to allow efficient thermalization of the inflaton energy density. Models satisfying these criteria do not arise naturally in particle physics; they have to be constructed ad hoc.

A less commonly discussed alternative is that the entropy in the matter fields comes from gravitational particle production at the end of inflation. If Since the inflaton does not interact with matter, and its energy density can roll monotonically toward zero, it is a candidate for a present-day effective cosmological constant. This picture merits broader discussion. To this end we present a specific example that we believe satisfies the main observational constraints. Our choice of the inflaton potential is presented in the next section. The results of a numerical

\footnotetext{
${ }^{1}$ In another terminology, matter fields end up in squeezed states after inflation [1].
}

integration of the evolution from inflation to the present are shown in Figure 1.

Ford [2] first investigated gravitational particle production at the end of inflation, and pointed out that particles created this way could account for the entropy of the present universe. Inflation would be followed by a period of expansion dominated by the energy density $\rho_{\phi}$ of the inflaton. Ford noted that if the kinetic part $\dot{\phi}^{2} / 2$ were dominant the energy density would decay with the expansion factor $a(t)$ as $\rho_{\phi} \propto a^{-6}$, so the expansion could become radiation-dominated before light element production. Spokoiny [3] added the idea that the inflaton could end up as the scalar field that Peebles and Ratra [4] proposed serves as an effective present-day cosmological constant. Spokoiny [3] presented examples of potentials $V(\phi)$ in which there is a transition from inflation to kinetic energy-dominated expansion, and he considered the conditions under which the expansion later is dominated again by the inflaton. Since gravity waves are produced by inflation with the same energy density per component as the matter fields, the entropy at very high redshift must be distributed among enough matter field components that gravitational waves do not make a significant contribution to the expansion rate during light element production. We discuss this condition in $\S \mathrm{VI}$, along with the possible detection of the gravitational waves by future detectors. Other possible connections to observations include the effect of the kinetic energy-dominated expansion on relict particle abundances, as discussed by Kamionkowski and Turner 5, and on baryogenesis, as considered by Joyce [6] and Joyce and Prokopec [7]. There may also be a conceptual value to the postulate that the inflaton potential resides in a sector that is not coupled to matter fields, and so may assume a simple and possibly "natural" form.

Terminology might be mentioned. Spokoiny [3] calls the kinetic energy-dominated period "deflationary;" Joyce [6] prefers "kination." Caldwell, Dave, and Steinhardt 86 propose the name "quintessence" for a field that acts like Einstein's cosmological constant $\Lambda$. Since the model under discussion reduces the role of the inflaton to the essential operation of driving inflation, and adds the possibility that the inflaton ends up as quintessence, 
we call the picture "quintessential inflation."

\section{THE MODEL}

We consider inflation driven by a single scalar inflaton field $\phi$ that interacts only with gravity and itself by the potential $V(\phi)$. The matter fields are scalar, spinor and gauge fields of some grand unified theory, and are supposed to be in their vacuum states during inflation.

The homogeneous part of the inflaton field satisfies

$$
\frac{d^{2} \phi}{d t^{2}}+3 \frac{\dot{a}}{a} \frac{d \phi}{d t}=-\frac{d V}{d \phi},
$$

where the expansion rate is

$$
H^{2}=(\dot{a} / a)^{2}=\left(\rho_{\phi}+\rho_{m}\right) / m_{\mathrm{pl}}^{2},
$$

and the Planck mass is written as

$$
m_{\mathrm{pl}}=(8 \pi G / 3)^{-1 / 2}=4.2 \times 10^{18} \mathrm{GeV},
$$

with $\hbar=1=c$. The energy density in the inflaton is

$$
\rho_{\phi}=\dot{\phi}^{2} / 2+V(\phi)
$$

and $\rho_{m}(t)$ is the matter energy density after inflation.

We adopt the inflaton potential

$$
\begin{aligned}
V & =\lambda\left(\phi^{4}+M^{4}\right) \quad \text { for } \quad \phi<0, \\
& =\frac{\lambda M^{8}}{\phi^{4}+M^{4}} \quad \text { for } \quad \phi \geq 0
\end{aligned}
$$

At $-\phi \gg M$ this is a "chaotic" inflation potential [10]; at $\phi \gg M$ it is a "quintessence" form of the type considered in Refs. [4], [8], [9]. We adopt

$$
\lambda=1 \times 10^{-14},
$$

from the condition that present-day large-scale structure grows from quantum fluctuations frozen into $\phi$ during inflation. The following sections establish bounds on the constant energy parameter $M$. The example in Figure 1 assumes the present density parameter in matter is $\Omega_{m} \sim$ 0.3 , with $\Omega_{\phi}=1-\Omega_{m} \sim 0.7$ in the inflaton. Then with the model for matter and radiation in $\S \S \mathrm{V}$ and VI,

$$
M=8 \times 10^{5} \mathrm{GeV} .
$$

\footnotetext{
${ }^{2}$ On scales of astronomical interest the only difference from "chaotic" inflation with $V(\phi)=\lambda \phi^{4}$ is the increased expansion factor to the end of inflation. Since the adibatic density fluctuations from the frozen inflaton depend on the logarithm of the expansion factor [10], the increased expansion is not significant. In conventional inflation the value of $\lambda$ from the COBE normalization 11 is three times the rounded value in Eq. (6). Again, the precise value of $\lambda$ is not important.
}

\section{KINETIC ENERGY-DOMINATED EXPANSION}

We assume the inflaton starts at $\phi \ll-m_{\mathrm{pl}}$ and rolls toward zero. Inflation ends at $\phi \sim-m_{\mathrm{pl}}$ when a substantial part of the potential energy has turned into the kinetic energy of the inflaton. This is followed by a kinetic energy-dominated peri od where $V(\phi)$ and the matter energy density terms in Eqs. (11) to (4) are negligible. Here the solution to Eq. (11) is $\dot{\phi} \propto a^{-3}$, and the energy density is

$$
\rho_{\phi} \approx \dot{\phi}^{2} / 2 \sim \lambda m_{\mathrm{pl}}^{4}\left(a_{x} / a\right)^{6}
$$

where $a_{x}$ is the expansion factor at the end of inflation. This expression in the expansion rate Eq. (2) yields

$$
a \sim t^{1 / 3}, \quad \phi=2^{1 / 2} m_{\mathrm{pl}} \ln \left(a / a_{x}\right) .
$$

The central point, as shown by Ford [2] and Spokoiny [3], is that the inflaton energy density decreases faster than that of radiation, so if the potential remains negligible the energy of the particles produced at the end of inflation eventu ally dominates. Our potential decreases only logarithmically with $a, V \sim \lambda M^{8} m_{\mathrm{pl}}^{-4}\left[\ln \left(a / a_{x}\right)\right]^{-4}$, so if the radiation remains subdominant $V(\phi)$ approaches the kinetic energy density at scale factor $a_{*}$ given by

$$
\frac{a_{*}}{a_{x}} \sim\left(\frac{m_{\mathrm{pl}}}{M}\right)^{4 / 3} \ln ^{2 / 3}\left(\frac{m_{\mathrm{pl}}}{M}\right) .
$$

The expansion then enters inflation from which it never recovers. Our model has a chance to work ${ }^{3}$ only if the matter energy density $\rho_{m}\left(a_{x}\right)$ at the end of inflation is large enough to dominate the inflaton energy density at $a=a_{r}<a_{*}$. We now turn to the estimate of $\rho_{m}$.

\section{PARTICLE CREATION AND THE RADIATION ERA}

Particle creation at the end of inflation can be studied by the standard methods of quantum field theory in curved spacetime [12]. For a massless scalar field $\chi$ described by the Lagrangian

$$
L=\frac{1}{2}\left(\partial_{\mu} \chi\right)^{2}-\frac{1}{2} \xi \mathcal{R} \chi^{2}
$$

\footnotetext{
${ }^{3}$ One sees from Eqs. (8) and (9) that $\rho_{\phi}$ after inflation remains dominated by the kinetic energy part until $\rho_{m}$ becomes important if the potential varies as rapidly as $V \propto e^{-\phi / \phi_{c}}$ with $\phi_{c}<m_{\mathrm{pl}} /(3 \sqrt{2})$. In our example the exponential variation of $V$ with $\phi$ is replaced by the rapid functional change of $V$ at $\phi \sim 0$ because of the small value of $M / m_{\mathrm{pl}}$.
} 
where $\mathcal{R}$ is the scalar curvature, this has been done by Ford [2] in the limit of nearly conformal coupling, $\mid \xi-$ $\frac{1}{6} \mid \ll 1$. He assumes that during inflation the spacetime is close to de Sitter, and that inflation is immediately followed by radiation-dominated expansion. His result for the energy density of the created particles is

$$
\rho_{m}=R H_{x}^{4}\left(a_{x} / a\right)^{4}
$$

where $H_{x}$ is the Hubble parameter, inflation ends at $a=$ $a_{x}$, and the numerical factor isf

$$
R \sim 10^{-2}(1-6 \xi)^{2}
$$

Ford's analysis [2] was extended to arbitrary values of $\xi$ 13. and to arbitrary power-law expansion after inflation 15. In all cases $R \sim 10^{-2}$. We assume minimal coupling, $\xi=0$, and adopt $R=0.01$ per scalar field component.

Free massless spinor and gauge fields are conformallyinvariant, so their contribution to $\rho_{m}$ is suppressed relative to scalar fields. 5 Thus in a simple model of gravitational production of quanta with negligible rest mass the matter energy density is given by Eq. (12) with

$$
R \sim 10^{-2} N_{s}
$$

where $N_{s}$ is the number of scalar fields.

We consider next the thermalization of $\rho_{m}$. For $\lambda=$ $10^{-14}$ the Hubble parameter at the end of inflation is

$$
H_{x} \sim \lambda^{1 / 2} m_{\mathrm{pl}} \sim 10^{12} \mathrm{GeV}
$$

at the earliest filled squares in Figure 1. The created particles have typical energy $\epsilon \sim H_{x}\left(a_{x} / a\right)$ and number density $n \sim R \epsilon^{3}$. As Spokoiny [3] noted, the particles interact by the exchange of gauge bosons and establish thermal equilibrium among the fermions and gauge bosons when the interaction rate $n \sigma$ becomes comparable to the expansion rate $H$. Here, $\sigma \sim \alpha^{2} \epsilon^{-2}$ and $\alpha \sim 0.1-0.01$ is the gauge coupling constant. With $H \sim H_{x}\left(a_{x} / a\right)^{3}$, thermalization occurs at

$$
a_{\mathrm{th}} / a_{x} \sim \alpha^{-1} R^{-1 / 2} \sim\left(10^{2}-10^{3}\right) N_{s}^{-1 / 2},
$$

\footnotetext{
${ }^{4}$ The "sudden" approximation, in which the de Sitter line element is matched to the radiation-dominated one, is adequate for modes with wave numbers $k \ll H_{x}$ and can be expected to give correct orders of magnitude for $k \sim H_{x}$. Particle production is strongly suppressed for $k \gg H_{x}$. The main contribution to $\rho_{m}$ is given by the modes with $k \sim H_{x}$, and thus Eq. (13) can be regarded only as an order-of-magnitude estimate.

${ }^{5}$ In the case of interacting fields, particle production can occur even if the field equations are conformally invariant. This is due to the conformal anomaly of the quantum theory [14.
}

only a few orders of magnitude expansion from the end of inflation, at temperature

$$
T_{\mathrm{th}} \sim \rho_{m}^{1 / 4}\left(a_{\mathrm{th}}\right) \sim R^{3 / 4} \alpha H_{x} \sim 10^{9} N_{s}^{3 / 4} \mathrm{GeV}
$$

During kinetic-energy dominated expansion the ratio of energy densities in matter and the inflaton is (Eqs. 8 and 12)

$$
\rho_{m} / \rho_{\phi} \sim \lambda R\left(a / a_{x}\right)^{2}
$$

Radiation-dominated expansion thus begins at expansion factor

$$
a_{r} / a_{x} \sim(\lambda R)^{-1 / 2}
$$

at the second earliest filled squares in Figure 1. We require $a_{r}<a_{*}$, where $a_{*}$ is given by Eq.(10). With $R \sim 0.01-1$, this gives the condition

$$
M<\left(10^{13}-10^{14}\right) \mathrm{GeV}
$$

well above the value for quintessence (Eq. 7). The temperature at the start of radiation-dominated expansion is, from Eq. (14),

$$
T_{r} \sim \rho_{m}^{1 / 4}\left(a_{r}\right) \sim \lambda R^{3 / 4} m_{\mathrm{pl}} \sim 10^{3} N_{s}^{3 / 4} \mathrm{GeV} .
$$

\section{EVOLUTION THROUGH THE RADIATION ERA TO THE PRESENT}

At $a \sim a_{r}$ the inflaton satisfies

$$
\phi_{r} \sim 2^{1 / 2} m_{\mathrm{pl}} \ln (\lambda R)^{-1 / 2}, \quad \dot{\phi}_{r} \sim R^{3 / 2} \lambda^{2} m_{\mathrm{pl}}^{2} .
$$

In an extended period after $a_{r}$ the potential gradient term in the field equation (11) for $\phi$ is negligible and the solution for radiation-dominated expansion with the initial conditions (22) is $\phi=A-B\left(t_{r} / t\right)^{1 / 2}$, with $A \approx \phi_{r}$ and $B \sim m_{p l}$. That is, $\phi$ is increasing quite slowly. at During matter-dominated expansion there is a stable increasing solution,

$$
\phi=k \lambda^{1 / 6} M^{4 / 3} t^{1 / 3},
$$

where $k=(72 / 5)^{1 / 6}$ in the radiation era and $k=3^{1 / 3}$ in the nonrelativistic matter era. The inflaton remains close to constant at $\phi \sim \phi_{r}$ until this solution becomes comparable to $\phi_{r}$, at

$$
t_{a} \sim \lambda^{-1 / 2} m_{p l}^{3} M^{-4}\left[\ln (\lambda R)^{-1 / 2}\right]^{3} \sim 10^{11} \mathrm{y},
$$

for our parameters. Since this is much greater than the present expansion time $\rho_{\phi}$ is nearly constant from the end of kinetic-energy dominated expansion to the present, as in Figure 1. The present energy density in the inflaton thus is $\rho_{\phi} \sim V\left(\phi_{r}\right)$, and the condition that this is comparable to the total present energy density is 


$$
M \sim \lambda^{-1 / 8} m_{\mathrm{pl}}^{3 / 4} H_{o}^{1 / 4}\left[\ln (\lambda R)^{-1}\right]^{1 / 2} \sim 10^{6} \mathrm{GeV},
$$

close to the numerical result (Eq. 7).

When the energy density $\rho_{\phi}$ in the inflaton remains subdominant to $a>a_{r}$ the expansion factor from the end of inflation to the time when $\rho_{\phi}$ reaches the nearly constant value $V\left(\phi_{r}\right)$ follows as in Eq. (10),

$$
a_{*} / a_{x} \sim\left(m_{\mathrm{pl}} \phi_{r}\right)^{2 / 3} / M^{4 / 3} .
$$

This is $a_{*} / a_{x} \sim 10^{18}$ for our parameters.

It might be noted that the model is not sensitive to the power law index in the potential after inflation. The potential in Eq. (5) at $\phi>0$ may be generalized to

$$
V=\frac{\lambda M^{4}}{1+(\phi / M)^{\alpha}} .
$$

Since $V$ at $a_{*}<a<a_{o}$ is determined by the density parameter $\Omega_{\phi}=1-\Omega_{m}=0.7$ and Hubble's constant, which fix the present value of $\rho_{\phi}$, the combination $\lambda M^{4+\alpha} / \phi_{r}^{\alpha}$ is independent of $\alpha$. For $\alpha=4$ and $\lambda=1 \times 10^{-14}$ we require $M / m_{\mathrm{pl}}=2 \times 10^{-13}$ to agree with $\Omega_{\phi}$. For the same value of $\lambda$ and $\alpha=6$ we need $M / m_{\mathrm{pl}}=1.3 \times 10^{-10}$, and, for $\alpha=2, M / m_{\mathrm{pl}}=4 \times 10^{-18}$. For this range of values of the power law index $\alpha$ and the indicated scaling of $M$ the evolution to the present is very similar to what is shown in Figure 1.

At $a>a_{0}$, when the inflaton energy dominates, the inflationary expansion can be described in the slow roll approximation, which neglects $\ddot{\phi}$ in Eq. (1) and the kinetic energy density term in Eq. (4). This gives

$$
\begin{aligned}
\phi & \approx \phi_{r}\left(1+C H_{0} t\right)^{1 / 4}, \\
a & \propto \exp \left[\frac{2}{C}\left(1+C H_{0} t\right)^{1 / 2}\right],
\end{aligned}
$$

where $C=16 m_{p l}^{2} / 3 \phi_{r}^{2} \approx 0.01$ and we have used $H_{o} \approx$ $V\left(\phi_{r}\right) / m_{p l}^{2}$ for the present expansion rate. The expansion rate remains nearly constant and the growth of $\phi$ is approximately linear in $t$ until the time $t_{b} \sim 1 / C H_{o} \sim$ $10^{12} \mathrm{y}$.

\section{GRAVITATIONAL WAVES}

Overproduction of gravitational waves is one of the dangers to be watched for in quintessential inflation. Gravitons are described by the same equation as a minimally coupled scalar field, so the energy density of gravitons at the end of inflation is twice that of a single scalar field because of the two graviton polarization states [16. Quanta of the inflaton field $\phi$ will also be produced, so the ratio of energy densities in the graviton-inflaton quanta and the matter density (Eqs. 12, 14) at the end of inflation is

$$
r_{x}=\frac{\rho_{g i}}{\rho_{m}}\left(a \sim a_{x}\right) \sim \frac{3}{N_{s}} .
$$

The annihilation of field quanta as the temperature falls below the mass conserves entropy (apart from the neutrinos at $z \sim 10^{10}$ ), so the matter temperature varies as $T_{m} \propto a^{-1} \mathcal{N}^{-1 / 3}$, where $\mathcal{N}(T)$ is the effective number of spin degrees of freedom in equilibrium at temperature $T$. Thus the mass fraction in graviton and inflaton quanta immediately before electron-positron annihilation is

$$
r_{n}=\frac{\rho_{g i}}{\rho_{m}}\left(a \sim a_{n}\right) \approx \frac{3}{N_{s}}\left(\frac{\mathcal{N}_{n}}{\mathcal{N}_{\text {th }}}\right)^{1 / 3}<0.07 .
$$

At matter thermalization following the end of inflation, $\mathcal{N}_{\text {th }} \sim 10^{2}-10^{3}$. When light element production commences at $z \sim 10^{10}, \mathcal{N}_{n}=10.75$ (in radiation with $\mathcal{N}=2$, electron-positron pairs, and three families of neutrinos). One extra neutrino family would increase the mass density by $16 \%$. As indicated in Eq. (30), the standard model for the light elements requires that the gravitons add significantly less than this [16].

In a minimal GUT, the only light particles with masses below $H_{x}$ are those of the standard model. Here $N_{s}=4$ (the electroweak Higgs doublet), $\mathcal{N}_{t h} \sim 10^{2}$, and $r_{n} \sim$ 0.3 , the equivalent of two new neutrino families. This is unacceptable within the standard model. A model with an intermediate symmetry breaking scale at energy $\lesssim H_{x}$ can give a substantially larger $N_{s}$. Alternatively, in supersymmetric theories there is a complex scalar field for each chiral quark or lepton. For the minimal model this gives $N_{s}=104$ and $r_{n} \sim 0.01$, well within the bound in Eq. (30).

The spectrum of gravitational waves differs from the usual inflation picture [16] because of the long kinetic energy-dominated period. Gravitational waves are produced with strain per logarithmic interval of frequency $h_{g} \sim H_{x} / m_{\mathrm{pl}}$ at Hubble length crossing during inflation. The amplitude is nearly constant $\mathrm{F}$ until the proper wavelength re-crosses the Hubble length at expansion parameter $a_{\omega}$, after which the amplitude decreases as $1 / a(t)$ to present value

$$
h_{g} \sim\left(H_{x} / m_{\mathrm{pl}}\right) a_{\omega} / a_{o}, \quad H\left(a_{\omega}\right) \sim \omega a_{o} / a_{\omega},
$$

at present expansion parameter $a_{o}$ and present proper frequency $\omega$. Waves that pass the Hubble length at the

\footnotetext{
${ }^{6}$ More accurately, when the proper wavelength is much larger than the Hubble length, and $a \propto t^{1 / 3}$ during kinetic energydominated expansion, the more rapidly growing solution to the field equation $\ddot{h}_{g}+3 \dot{h}_{g} \dot{a} / a=0$ is $h_{g}=\ln t$ [15]. Here we ignore this slow evolution. A more careful analysis by Giovannini [15] shows that it results in a logarithmic correction to the gravitational wave spectrum (34), $h_{g} \propto \omega^{-1 / 2} \ln \left(\omega_{x} / \omega\right)$.
} 
time of equality of mass densities in relativistic and nonrelativistic matter have present frequency

$$
\omega_{\text {eq }} \sim 3 \times 10^{-16} \Omega_{m} \mathrm{~Hz} .
$$

Waves that pass the Hubble length at the end of inflation and at the start of radiation-dominated expansion have present frequencies

$$
\begin{aligned}
\omega_{x} & \sim T_{o} R^{-1 / 4} \sim 10^{11} \mathrm{~Hz}, \\
\omega_{r} & \sim \lambda R^{3 / 4} T_{o} \sim 10^{-3} \mathrm{~Hz},
\end{aligned}
$$

for our parameters. The part of the gravitational wave spectrum that starts oscillating during kinetic energydominated expansion, at $\omega_{r}<\omega<\omega_{x}$, is 15]

$$
h_{g} \sim \frac{T_{o}^{3 / 2}}{R^{3 / 8} m_{\mathrm{pl}} \omega^{1 / 2}} \sim 10^{-24}\left(\frac{\omega_{r}}{\omega}\right)^{1 / 2} .
$$

Between $\omega_{r}$ and $\omega_{\text {eq }}$ the frequency dependence changes to $h_{g} \propto \omega^{-1}$, the same as usual inflation models.

The form of the gravitational wave spectrum at $\omega>\omega_{r}$ can serve as an observational signature of quintessential inflation. The signal in our example is too small to be detected by interferometric graviational wave detectors under construction, including LIGO and VIRGO, but may be within reach of future detectors.

\section{DISCUSSION}

The model meets several observational constraints. Primordial density fluctuations are essentially the same as in a chaotic inflation model [10], and lead to the adiabatic cold dark matter model for structure formation. This is viable but not yet shown to be unique. Perhaps topological defects that originate near the end of inflation, or the fields that produce the entropy in this model, also play a role in structure formation. The expansion becomes radiation-dominated at redshift $z \sim 10^{18}$, well before light element production, and the energy density in gravitational waves may be tolerable. Baryogenesis remains an open issue, as in conventional inflation [6], [7]. The model is adjusted to present-day density parameter $\Omega_{m}=0.3$. There is considerable evidence for this low value [17]. There is evidence for the zero space curvature required here and in usual inflation, from the redshift-magnitude relation for type Ia supernovae [18, [19]. This difficult measurement should be checked for consistency with other constraints on $\Lambda$. The reading of evidence from the anisotropy of the thermal cosmic background radiation (the CBR) on open vs. cosmologically flat models still is mixed [20], [21].

Our model fails to show the tracking of the inflaton and matter mass densities proposed by Zlatev, Wang and Steinhardt [9]. We have checked that in the range of values $2<\alpha<6$ for the potential $V \propto \phi^{-\alpha}$ (Eq. 27) well after inflation the energy density $\rho_{\phi}$ in the inflaton is quite close to constant over ten orders of magnitude of expansion centered on the present epoch. During this time the inflaton pressure is quite close to $-\rho_{\phi}$, so the field does not respond significantly to gravitational perturbations. Thus we suspect that, in contrast to the tracking case [22], 8], it will be difficult to find classical cosmological tests that distinguish our model from a Friedmann-Lemaître model with constant Lambda.

There are arguably unnatural features of our model. First, the model parameters have to be tuned to arrange inflaton domination at the present epoch. This problem is common to all cosmological scenarios with $\Omega_{m}<1$. Second, it is somewhat unnatural for a small mass $M \ll m_{p l}$ to appear in the potential of the inflaton field $\phi$ interacting only with gravity. Both problems can be addressed in models with an extra inflaton field $\chi$ in which $M$ is not a constant but a slowly varying function of $\chi$. Different parts of the universe would then thermalize with different values of $M$, and a low value of $M$ in our neighborhood could be selected by anthropic considerations. Regions with $M \gg 10^{6} \mathrm{GeV}$ would become inflaton-dominated much earlier, and galaxy formation is such regions would be suppressed. This is similar to the anthropic selection of the cosmological constant, as discussed in [23].

The quintessential inflation model simplifies the role of the inflaton by decoupling it from the matter. It remains to be seen whether this will aid the search for a believable physical basis for the inflaton.

\section{ACKNOWLEDGMENTS}

We are grateful for discussions with Massimo Giovannini, Alan Guth, Guy Moore, Paul Steinhardt, and Michael Turner. This work was supported in part at the Princeton Institute for Advanced Study by the Alfred P. Sloan Foundation and at Tufts University by the National Science Foundation.

[1] L.P. Grishchuk and Y.V. Sidorov, Phys. Rev. D 42, 3413 (1990).

[2] L. H. Ford, Phys. Rev. D 35, 2955 (1987).

[3] B. Spokoiny, Phys. Lett. B 315, 40 (1993).

[4] P. J. E. Peebles and B. Ratra, Astrophys. J. 352, L17 (1988).

[5] M. Kamionkowski and M. S. Turner, Phys. Rev. D 42, 3310 (1990).

[6] M. Joyce, Phys. Rev. D 55, 1875 (1997).

[7] M. Joyce and T. Prokopec, Phys. Rev. D 57, 6022 (1998). 
[8] R. R. Caldwell, R. Dave, and P. J. Steinhardt, Phys. Rev. Lett. 80, 1582 (1988).

[9] I. Zlatev, L. Wang, and P. J. Steinhardt (astro$\mathrm{ph} / 9807002$ ).

[10] For a review of inflation see, e.g., A. D. Linde, Particle Physics and Inflationary Cosmology (Harwood, Chur, 1990).

[11] H. Kurki-Suonio and G. J. Mathews, Phys. Rev. D 50, 5431 (1994).

[12] N. D. Birrell and P. C. W. Davies, Quantum fields in Curved Space (Cambridge University Press, Cambridge, 1982).

[13] T. Damour and A. Vilenkin, Phys. Rev. D 53, 2981 (1996).

[14] N. D. Birrell and P. C. W. Davies, Phys. Rev. D 22, 322 (1980).

[15] M. Giovannini, Phys. Rev. D 58, 083504 (1998)

[16] For a review of production mechanisms of and observational bounds on a stochastic gravitational wave background see, e.g., B. Allen, in Proceedings of the Les Houches School on Astrophysical Sources of Gravitational Waves, ed. by J. Marck and J. P. Lasota (Cambridge University Press, Cambridge, 1996).

[17] P.J.E. Peebles, Publ. Astron. Soc. Pacific, in press (1999); astro-ph/9810497.

[18] S. Perlmutter et al., LBNL-41801 preprint (1998).

[19] A. G. Reiss et al., Astrophys. J. 115 astro-ph/9805201) (1998).

[20] E. Gawiser and J. Silk, Science 280, 1405 (1998).

[21] K. M. Górski, B. Ratra, R. Stompor, N. Sugiyama, and A. J. Banday, Astrophys. J. Suppl. 114, 1 (1998).

[22] B. Ratra, B. and A. Quillen, MNRAS 259, 738 (1992).

[23] S. Weinberg, Phys. Rev. Lett. 59, 2607 (1987); A. Vilenkin, Phys. Rev. Lett. 74, 846 (1995); H. Martel, P. R. Shapiro and S. Weinberg, Ap. J. 492, 29 (1998).

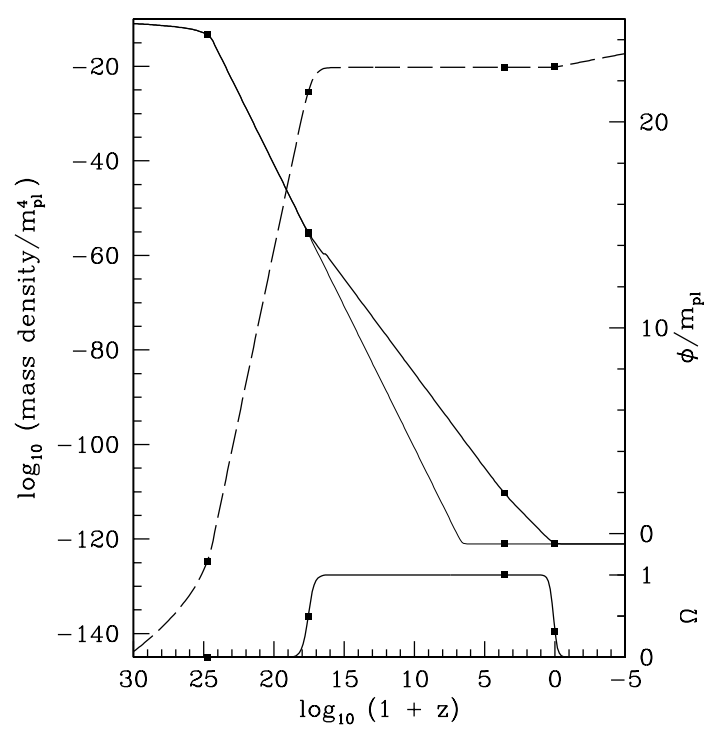

FIG. 1. Evolution in quintessential inflation. The total mass density is plotted as a heavy solid line, the lighter line is the mass density in the inflaton, and the dashed line is the inflaton field value. The bottom curve is the density parameter (the fractional contribution to the square of the Hubble parameter by the mass densities in relativistic and nonrelativistic matter). The end of inflation, marked by the earliest (left-hand) filled squares, is defined by the maximum of $a(t) H(t)$ (the minimum value of the comoving Hubble length). Radiation-dominated expansion commences at the next squares. The next square indicates the epoch of equal mass densities in radiation and nonrelativistic matter. The last squares mark the present epoch at radiation temperature $T \sim 3^{\circ} \mathrm{K}$. The parameter $M$ in the potential (Eqs. 5 to 7 ) is chosen so the present value of the density parameter in matter is $\Omega_{m}=0.3$. The model for the matter assumes $R=1(\mathrm{Eq}$ 14) and $\mathcal{N}_{\mathrm{th}}=1000$ effective scalar fields at first thermalization. Annihilation of the extra fields at $T=1000 \mathrm{GeV}$ causes the step in the mass density at redshift $z \sim 10^{16}$ (Eq. 30). 